\title{
Age Related Disparities in Colorectal Cancer Patients
}

Aliya Ishaq

Specialist general surgery and lecturer general surgery Dubai Medical College Dubai UAE.

Corresponding Author: Aliya Ishaq, Specialist general surgery and lecturer general surgery Dubai Medical College Dubai UAE.

Received date: July 24, 2021; Accepted date: September 04, 2021; Published date: September 10, 2021

Citation: A Ishaq. (2021) Age Related Disparities in Colorectal Cancer Patients. Journal of Clinical and Laboratory Research. 3(3); DOI:10.31579/2768-0487/064

Copyright: (C2021 Aliya Ishaq. This is an open-access article distributed under the terms of the Creative Commons Attribution License, which permits unrestricted use, distribution, and reproduction in any medium, provided the original author and source are credited.

\begin{abstract}
:
Background: There is an evident change in the colorectal cancer demographic over the period. This change is more marked in the age distribution and location of the tumor. It has practical implications, in regards to develop cancer awareness programs and screening protocols. Keeping in view that Pakistan is one of the countries with a high number of the young population this study is carried out to make a comparative analysis of this trend in our population.

Material and methods: Colorectal cancer patients presented in Sindh Institute of urology and transplantation from January 2011 till December 2020 was reviewed retrospectively. All patients were divided into two groups, Group A young age population and Group B old age population. Subgroup analysis of study period was performed to check the progressive change in the trend of stage and clinical characteristics of colorectal cancer patients. Data reviewed from the patient's files and collected as per Proforma requirement.

Result: Total of 612 patients with colorectal cancer presented between 2011 till 2020.Among these patients 243 (39.7\%) presented between January 2011 till December 2015. Patients age 50 years and younger were 410 (66.8\%). Results showed a statistically significant association between and patient's age and location of tumor such that left-sided colonic cancer and rectal cancer were more common in the young population. Subgroup analysis according to the study period showed that there is a change in the trend of disease presentation. Right-sided colonic cancer presentation decreased in the younger population over the period while simultaneously leftsided colonic cancer and rectal cancer presentation increased.
\end{abstract}

Conclusion: The incidence of left-sided colonic and rectal cancer has been increased in the younger population over the specified period while there was no association between right-sided colon cancer and age noticed.

Key words: colorectal cancer; right-sided colon; left-sided colon

\section{Background:}

The demographics of Colorectal Cancer (CRC) are changing. The two aspects demonstrating the most remarkable change include the age distribution and location of the tumor in the large bowel. Overall, CRC is the most common malignancy of the gastrointestinal tract, the 3rd most common cancer, and the 4th leading cause of death by cancer $[1,2,3]$. With regards to the age distribution of CRC, between 2009 and 2013 colorectal cancer incidence decreased by $4.6 \%$ per year in individuals aged 65 years and older and increased in individuals younger than 50 years by $1.6 \%$ per year $[1,2,3]$. This decrease in incidence among individuals older than 55 years of age is largely attributed to populationbased CRC screening recommendations in place since the 1980s $[1,2,3$, 4].

The increasing incidence among patients younger than 54 years of age also shows disparity related to location. So the incidence of rectal cancer has nearly quadrupled over the past few decades compared with colon cancer (41.5\% v 9.8\%, respectively) [1]. Subset analysis demonstrates an even finer-print regarding location-based incidence, among younger patients. So, for colon cancer, the adjusted incidence rates increased annually over roughly the past four decades by $2.4 \%$ in those aged 20 to 29 years, by $1.0 \%$ in those aged 30 to 39 years, and by $1.3 \%$ in those aged 40 to 49 years; for rectal cancer, the adjusted incidence rates have increased by $3.2 \%$ in those age $20-39$ years and by $2.3 \%$ in those age 40 49 years [4]

Interestingly, the reduction in the incidence of colorectal cancer among older individuals, as explored earlier, is largely for distally located cancer, again demonstrating that the colorectal cancer incidence is also influenced by the location of tumor $[1,2,3]$.

This changing epidemiology of CRC has practical implications, in particular for age to start screening. Recently, the US Preventive Services Task Force has recommended starting CRC screening at age 45 years instead of years [7]. However, previous studies have described a wide variation of this frequency worldwide, with the highest variance, found in the Asian population [1,2]. Taking this into account and the dearth of related data in Pakistan, this study is being carried out to make a comparative analysis of these trends in CRC incidence in our population. This is particularly important because Pakistan has one of the youngest 
populations in the world, and with the increasing incidence of CRC in this age group, appropriate allocation of resources is warranted.

\section{Rationale of study:}

Despite informal observation by oncology professionals of shifting the age of onset of CRC to younger individuals and noting distinctive attributes of the disease in younger patients, few studies have formally documented this trend- even fewer in Pakistan. This practice-changing information needs more evidence to change attitudes and guidelines. This study is an effort in this regard.

\section{Objectives:}

1. To compare the proportion of diagnosed colorectal cancer between younger (less than and equal to 50 years) and older population (aged 51 years and above) over two consecutive periods spanning from January 2011- December 2015 and January 2016- December 2020.

2. To compare the proportional anatomical distribution of colorectal cancer between right-sided and left-sided tumors according to age group (younger and older, as defined above) over two consecutive periods spanning from January 2011- December 2015 and January 2016December 2020.

\section{Material and Methods:}

A retrospective study was conducted in the Sindh Institute of urology and transplantation GI surgery department on patients diagnosed case of colorectal cancer. All patients received in Hanifa Suleiman Oncology center fulfilling inclusion criteria from January 2011 till December 2020 enrolled in the study. The clinical record of all patients was retrieved from the Hanifa Suleiman Oncology clinic registry, GI clinic patient's registry, histopathology department, and hospital Record room. Variables mentioned in the questionnaire were all collected and stored with a given MR number maintaining data security and confidentiality. Approval for the study and exemption letter was obtained from Institutional Review Board (IRB) and ethical review committee (ERC). All variables mentioned in the questionnaire will be collected from the patient's files including age, Gender, endoscopy (colonoscopy / Sigmoidoscopy) findings, Histological Type, Grade, Site of Tumor. For data analysis, all patients were divided into two groups, Group A young age population (age between 18-50 years) Group B old age population (age 51 years and Onward). The young age population further subdivided into three groups, A1 (age 18-30 years), A2 (age 31-40 years), A3 (age 41-50 years) while the old age population also subdivided into three groups B1 (age 51-60 years), B2 (age 61-70 years) and B3 (age 71yeas onward). Subgroup analysis of study period (To check the progressive change in the trend of CRC in young patients) from January 2011 - December 2015 first period while from January 2016 - December 2020 second period. Subgroup analysis of study period (To check the progressive change in the trend of
CRC in elder patients) from January 2011 - December 2015 first period while from January 2016 - December 2020 second period. Left-sided colonic Cancer includes cancer of descending Colon, distal 1/3rd of transverse Colon Cancer + sigmoid colon. Right-sided colonic Cancer includes cancers of ascending colon + proximal 2/3rd of transverse colon cancer. Rectal Cancer includes cancer anywhere from the rectosigmoid junction up to the anal verge.

\section{Statistical Analysis:}

All data collected were analyzed using SPSS version 23.0. Frequencies and percentages were computed for categorical variables while mean \pm standard deviation for quantitative variables. Chi-square or Fisher's Exact Test was used to determining the association between groups. An unadjusted odds ratio with a $95 \%$ confidence interval was employed to assess the direction and magnitude of association between variables. Significance based on $\mathrm{p}$-value $<0.05$.

\section{Result:}

During this study period between 2011 till 2020, 612 patients presented with the diagnosis of colorectal cancer in our institute. Among these patients 243 (39.7\%) presented between January 2011 till December 2015 while $369(60.3 \%)$ presented between January 2016 till December 2020. Patients age 50 years and younger were $410(66.8 \%)$ while 202 patients $(32.2 \%)$ were 51 years or above. The mean age of patients at the time of presentation was $44.46+/-15.36$ years. Out of total $386(63.1 \%)$ were male. At the time of initial presentation, 80 patients $(13.1 \%)$ were diagnosed to have right colonic cancer, 96patients $(15.7 \%)$ had left-sided colonic cancer and 436 patients $(71.2 \%)$ had rectal cancer.

Results showed a statistically significant association between the patient's age and the location of the tumor. Left-sided colonic cancer and rectal cancer were more common in the young age population $80.2 \%$ and $66.7 \%$ as compared to the old age group $19.8 \%$ and $33.2 \%$ respectively. However, there is no statistical significance noted in right-sided colonic cancer between the two groups. Subgroup analysis based on age also showed similar results. When subgroup analysis is reviewed based on the study period results showed that there is a change in the trend of disease presentation. Right-sided colonic cancer decreased in the younger population over the period while simultaneously left-sided colonic cancer and rectal cancer increased with statistically significant results. On the other hand, no change in disease pattern was observed for right and leftsided colon cancer in the elderly population while rectal cancer presentation increased in the elderly age group over the period with a statistically significant P-Value.

Subgroup analyses based on age conclude that this change in the trend of disease, presentation is more marked between 31 till 60 years of age with statistically significant results.

\begin{tabular}{|c|c|c|c|c|}
\hline \multirow[b]{2}{*}{ AGE } & \multicolumn{3}{|c|}{$\begin{array}{l}\text { TUMOUR LOCATION } \\
\end{array}$} & \multirow[b]{2}{*}{ P-VALUE } \\
\hline & RIGHT COLON & LEFT COLON & RECTUM & \\
\hline$\leq 50$ YEARS & $40(50 \%)$ & $77(80.2 \%)$ & $292(66.7 \%)$ & \multirow[b]{2}{*}{0.000} \\
\hline$\geq 51$ YEARS & $40(50 \%)$ & $19(19.8 \%)$ & $144(33.2)$ & \\
\hline \multicolumn{5}{|c|}{ SUB GROUP ANALYSIS } \\
\hline $18-30$ YEARS & $8(20.0 \%)$ & $19(24.6 \%)$ & $113(38.6 \%)$ & \multirow{3}{*}{0.006} \\
\hline $31-40$ YEARS & $14(35.0 \%)$ & $25(32.4 \%)$ & $103(35.2 \%)$ & \\
\hline $41-50$ YEARS & $18(45.0 \%)$ & $33(42.8 \%)$ & $76(26.0 \%)$ & \\
\hline $51-60$ YEARS & $27(69.2 \%)$ & $6(30.0 \%)$ & $75(51.7 \%)$ & \multirow{3}{*}{0.057} \\
\hline $61-70$ YEARS & $10(23.1 \%)$ & $10(50.0 \%)$ & $44(30.8 \%)$ & \\
\hline 71 -- ONWARD & $3(7.7 \%)$ & $320.0(\%)$ & $25(17.5(\%)$ & \\
\hline ALL AGES & $80(100 \%)$ & $96(100 \%)$ & $436(100 \%)$ & - \\
\hline
\end{tabular}

Table 1: Age and Tumor Location Association. 


\begin{tabular}{|c|c|c|c|c|c|}
\hline \multirow[t]{2}{*}{ AGE } & \multirow{2}{*}{$\begin{array}{l}\text { YEAR OF } \\
\text { DIAGNOSIS }\end{array}$} & \multicolumn{3}{|c|}{ TUMOUR LOCATION } & \multirow[t]{2}{*}{ P-VALUE } \\
\hline & & RIGHT COLON & LEFT COLON & RECTUM & \\
\hline \multirow[t]{2}{*}{$\leq 50$ YEARS } & $2011--2015$ & $31(77.5 \%)$ & $27(35.0 \%)$ & $109(37.2 \%)$ & \multirow[b]{2}{*}{0.000} \\
\hline & $2016--2020$ & $9(22.5 \%)$ & $50(64.9 \%)$ & $183(62.6 \%)$ & \\
\hline \multirow[t]{2}{*}{$\geq 51$ YEARS } & 2011--2015 & $21(52.5 \%)$ & $11(57.8 \%)$ & $44(30.5 \%)$ & \multirow[b]{2}{*}{0.006} \\
\hline & $2016--2020$ & $19(47.5 \%)$ & $8(42.1 \%)$ & $100(69.4 \%)$ & \\
\hline \multirow[t]{2}{*}{ 18-30 YEARS } & 2011--2015 & $5(62.5 \%)$ & $5(26.3 \%)$ & $44(38.9 \%)$ & \multirow[t]{2}{*}{0.208} \\
\hline & 2016--2020 & $3(37.5 \%)$ & $14(73.7 \%)$ & $69(61.1 \%)$ & \\
\hline \multirow[t]{2}{*}{31 - 40 YEARS } & 2011--2015 & $12(85.7 \%)$ & $10(40.0 \%)$ & $38(36.9 \%)$ & \multirow[t]{2}{*}{0.002} \\
\hline & $2016--2020$ & $2(14.3 \%)$ & $15(60.0 \%)$ & $65(63.1 \%)$ & \\
\hline \multirow[t]{2}{*}{41 - 50 YEARS } & 2011--2015 & $15(78.9 \%)$ & $12(37.5 \%)$ & $28(36.4 \%)$ & \multirow[t]{2}{*}{0.003} \\
\hline & 2016--2020 & $4(21.1 \%)$ & $20(62.5 \%)$ & $49(63.6 \%)$ & \\
\hline \multirow[t]{2}{*}{51 - 60 YEARS } & 2011--2015 & $14(51.9 \%)$ & $4(66.7 \%)$ & $22(29.7 \%)$ & \multirow[t]{2}{*}{0.039} \\
\hline & $2016--2020$ & $13(48.1 \&)$ & $2(33.3 \%)$ & $52(70.3 \%)$ & \\
\hline \multirow[t]{2}{*}{61 - 70 YEARS } & 2011--2015 & $5((55.6 \%)$ & $6(60.0 \%)$ & $16(36.4 \%)$ & \multirow[t]{2}{*}{0.279} \\
\hline & 2016--2020 & $4(44.4 \%)$ & $4(40.0 \%)$ & $28(63.4 \%)$ & \\
\hline \multirow{2}{*}{71 - ONWARD } & 2011--2015 & $1(33.3 \%)$ & $1(25.0 \%)$ & $5(20.0 \%)$ & \multirow[t]{2}{*}{0.859} \\
\hline & $2016--2020$ & $2(66.6 \%)$ & $3(75.0 \%)$ & $20(80.0 \%)$ & \\
\hline \multirow[t]{2}{*}{ ALL AGES } & 2011--2015 & $52(65.0 \%)$ & $38(39.5 \%)$ & $153(35.0 \%)$ & \multirow[b]{2}{*}{0.000} \\
\hline & 2016--2020 & $28(35.05)$ & $58(60.4 \%)$ & $283(64.9 \%)$ & \\
\hline \multicolumn{2}{|l|}{ TOTAL } & $80(100 \%)$ & $96(100 \%)$ & $436(100 \%)$ & - \\
\hline
\end{tabular}

Table 2: Age and Tumour Location Association with Study Period.

\section{Discussion:}

Our study high lightened the fact that colorectal cancer incidence is increasing in the adult population over 10 years. We found that the incidence is shifted from $35 \%$ to $64.9 \%$ for left-sided colonic cancer while for rectal cancer the incidence is shifted from $37.2 \%$ to $62.6 \%$. Almost similar results have been coated in other studies $[4,5,6]$. The American cancer society recommended starting screening for colorectal cancer at the age of 45 years because of the increased burden of disease in their young population. This change in screening age is based on the assumption that screening this age group may produce preventive effects due to early detection of disease and hence prompt management [7].

Our study results are similar to that of American data for colorectal cancer and even younger population 30 to 50 years age is at risk in Pakistan to develop colorectal cancer. Therefore considering screening endoscopy and at least Sigmoidoscopy for this population will be a great effort to diagnose the disease at an early stage with possibly better chances of treatment and long-term survival. However, there is no proper screening protocol for colorectal cancer in third world countries like Pakistan even for the old age population because of limited resources. For this reason, it is too early to use our data and support screening protocols in Pakistan but further studies on this issue are relevant which will help to monitor the trend of disease in the Pakistani population and to assess the need for adaptation of screening practice. Furthermore, we should study the underlying cause for colorectal cancer and identify the population at high risk and for the purpose first step is to make clinicians well aware of the fact that colorectal cancer is increasing in the young population quite rapidly and that sharp vigilance is needed in diagnosing the disease at the time of initial presentation. Awareness programs and dissemination of screening information for colorectal cancer have the potential to develop a better understanding of the signs and symptoms of the disease among the general population [3].

Our results support that screening endoscopy (colonoscopy/ Sigmoidoscopy) has preventive effects on colorectal cancer patients. Our study was also supported by the study conducted by Nancy et al who reported that Sigmoidoscopy reduces mortality in left-sided colon cancer in up to $70 \%$ of cases but because of inadequate coverage of the whole colon, it has no survival benefit in right-sided colon cancer cases. Based on these findings future trials involving colonoscopy versus
Sigmoidoscopy for right and left-sided colonic cancer will certainly produce reliable risk prediction [1].

Our study has certain limitations, it is a retrospective study and hence the data has potential bias. Because of the retrospective nature of the study, we are unable to properly assess personal and family history and evaluate familial adenomatous polyposis syndrome. As this is a single-centered study with a limited number of cases, this study is not representing the true population as well as there is a lack of a national cancer registry. In this study, we only observe the trend of the disease over a specified period and are not able to address other associations. However, this study is among the first to give an overview of colorectal cancer incidence in the younger population of Pakistan. The major strength of the study is that it will develop colorectal cancer awareness and screening information in the general population. Hence we favor the development of awareness programs, screening protocols, and at least flexible Sigmoidoscopy screening in the young population given the predilection for left-sided cancers in this age group.

\section{Conclusion:}

For all cancer sites combined, left-sided colonic and rectal cancer incidence increased in the younger population while no association between right-sided colon cancer and age was observed. Hence majors need to be taken in terms of awareness programs, screening protocols, and etiological research to elucidate causes for this trend which will help to reduce the number of colorectal cases in our young population.

\section{Acknowledgement:}

Special thanks to Dr Muhammad Arsalan Khan Consultant HBP surgeon Sindh Institute of Urology and Transplantation for his valuable input regarding motivation and support for writing this article.

\section{Conflict of interest:}

Nothing to disclose.

\section{References:}

1. Ansa BE, Coughlin SS, Alema-Mensah E, Smith SA. (2018). Evaluation of colorectal cancer incidence trends in the United States (2000-2014). J Clin Med. 7(2): 22. 
2. GLOBOCAN. (2018). Estimated number of colorectal cancer new cases in 2018, worldwide, both sexes, all ages Cancer Today.

3. Darren R. Brenner, Emily Heer, Liam Sutherland, Yibing Ruan, Jill Tinmouth, Steven J. Heitman, Robert J. (2019). Hilsden. National Trends in Colorectal Cancer Incidence Among Older and Younger Adults in Canada. JAMA Network Open. 2(7):198090.

4. Elizabeth M Ward, Recinda L Sherman, S Jane Henley, et al. (2019). Annual Report to the Nation on the Status of Cancer, Featuring Cancer in Men and Women Age 20-49 Years. J Natl Cancer Inst 111(12):1279-1297.

5. Song M, Giovannucci E. (2016). Preventable incidence and mortality of carcinoma associated with lifestyle factors among white adults in the United States. JAMA Oncol. 2: 1154-1161.

6. Siegel RL, Fedewa SA, Anderson WF, et al. (2017). Colorectal cancer incidence patterns in the United States, 1974-2013. J Natl Cancer Inst. 109(8): 322.

7. Kabat GC, Matthews CE, Kamensky V, Hollenbeck AR, Rohan TE. (2015). Adherence to cancer prevention guidelines and cancer incidence, cancer mortality, and total mortality: a prospective cohort study. Am J Clin Nutr. 101: 558-569.

8. US Preventive Services Task Force. (2021). Screening for Colorectal Cancer US Preventive Services Task Force recommendation Statement. JAMA. 325(19):1965-1977.

9. Andrew M. D. Wolf, Elizabeth T. H. Fontham, Timothy R. Church, et al. (2018). Colorectal Cancer Screening for Average-Risk Adults: 2018 Guideline Update From the American Cancer Society. Ca Cancer J Clin. 68: 250-281.

10. Lansdorp-Vogelaar1, L. von Karsa. (2012). European guidelines for quality assurance in colorectal cancer screening and diagnosis. First Edition Introduction. Endoscopy. 44 Suppl 3: $15-30$

11. Jacobs D, Zhu R, Luo J, et al. (2018). Defining early-onset colon and rectal cancers. Front Oncol 8:504.

12. Y. Nancy You, MD, Lucas D. Lee, Benjamin W. Deschner, et al. Colorectal Cancer in the Adolescent and Young Adult Population. JCO Oncol Pract 16:19-27.

13. Yeo H, Betel D, Abelson JS, et al. (2017). Early-onset colorectal cancer is distinct from traditional colorectal cancer. Clin Colorectal Cancer 16:293-299.

14. Joana Sofia Gomes Pestanaa, Sandra Fátima Fernandes Martin. (2016). Colorectal cancer: comparative analysis of clinical and pathological characteristics in patients aged above and below 45 years of age and impact on prognosis. J Coloproctol (Rio J). 3 6(4):196-202.

15. She K, Wang H, Chen J, Mar H, Chen C, Chiang F, et al. (2011). Colorectal cancer in younger than 30 years old group is not associated with poor prognosis. J Soc Colon Rectal Surgeon (Taiwan). 22: 93-98.

16. Elias F. Onyoh, Wen-Feng Hsu, Li-Chun Chang, Yi-Chia Lee, Ming-Shiang Wu, Han-Mo Chiu. (2019). The Rise of Colorectal Cancer in Asia: Epidemiology, Screening, and Management. Current Gastroenterology Reports 21:36.

17. Shaukat et al. (2021). ACG Clinical Guidelines: Colorectal Cancer Screening 2021 The American Journal of Gastroenterology: 116(3): 458-479.

18. Nancy N. Baxter, Joan L. Warren, Michael J. Barrett, Therese A, Stukel, V. Paul Doria-Rose. (2012). Association Between Colonoscopy and Colorectal Cancer Mortality in a US Cohort According to Site of Cancer and Colonoscopist Specialty J Clin Oncol. 30(21): 2664-2669.
This work is licensed under Creative Commons Attribution 4.0 License

To Submit Your Article Click Here: Submit Manuscript

DOI: $10.31579 / 2768-0487 / 039$
Ready to submit your research? Choose Auctores and benefit from:

fast, convenient online submission

$>$ rigorous peer review by experienced research in your field

$>$ rapid publication on acceptance

$>$ authors retain copyrights

$>$ unique DOI for all articles

$>$ immediate, unrestricted online access

At Auctores, research is always in progress.

Learn more auctoresonline.org/journals/journal-of-clinical-andlaboratory-research 